\title{
Vers un nouveau modèle de gouvernance de l'innovation pour les programmes de Défense et de Sécurité
}

L'apport de la dynamique de l'innovation ouverte et des communautés des logiciels « Open source »

Thomas Le Texier et David W. Versailles

\section{(2) OpenEdition}

\section{Journals}

Édition électronique

URL : http://journals.openedition.org/ei/245

DOI : $10.4000 /$ ei.245

ISSN : 2553-1891

Éditeur

Association Économie et Institutions

Édition imprimée

Date de publication : 30 octobre 2009

Pagination : 61-92

ISSN : 1775-2329

Référence électronique

Thomas Le Texier et David W. Versailles, « Vers un nouveau modèle de gouvernance de l'innovation pour les programmes de Défense et de Sécurité », Économie et institutions [En ligne], 12-13| 2009, mis en ligne le 31 janvier 2013, consulté le 19 avril 2019. URL : http://journals.openedition.org/ei/245 ; DOI : 10.4000/ei.245 


\title{
Vers un nouveau modèle de gouvernance de l'innovation pour les programmes de Défense et de Sécurité : L'apport de la dynamique de l'innovation ouverte et des communautés des logiciels "Open source " 1
}

\author{
Thomas LE TEXIER ${ }^{2}$ et David W. VERSAILLES ${ }^{3}$
}

\section{Introduction}

Les politiques publiques associées à la Défense découlent d'une mission régalienne. Longtemps associées à un cadre national, elles se repositionnent dans un cadre de coopération internationale ou européenne qui oblige à élargir le nombre des acteurs présents. La véritable révolution dans la gestion des questions industrielles associées aux programmes d'armement provient de l'ouverture des réseaux d'innovation à des acteurs originaux structurés sous des formes nouvelles. Le fonctionnement en interface avec des acteurs marchands (firmes intégrateurs de systèmes, fournisseurs des divers modules associés aux programmes d'armement) oblige à prendre en compte la dynamique économique associée aux stratégies de ces groupes industriels. L'asymétrie entre la stratégie des firmes et celle de l'Etat a conduit à des incompréhensions et à des impasses en matière de politiques publiques. Aujourd'hui, les choses se complexifient un peu plus loin, avec l'arrivée d'acteurs nonmarchands et communautaires dans les réseaux qui concourent aux programmes militaires. L'analyse se trouve alors confrontée aux

\footnotetext{
1 Les idées présentées dans cet article ont été développées pour la première fois lors de travaux conduits par les auteurs lorsqu'ils étaient en poste au sein du Centre de recherche de l'Armée de l'air (CReA). Les auteurs y ont organisé en avril 2008 un séminaire consacré aux logiciels open source dans le cadre des programmes de Sécurité et de Défense (FLOSS 1.5). Ils remercient les participants pour tous les échanges fructueux. Les auteurs tiennent à exprimer leur gratitude à Dominique Torre, Eric Darmon, Patrick Cohendet, Valérie Mérindol, et à deux référés anonymes, pour leurs commentaires sur des versions antérieures de cette recherche.

2 GREDEG - UMR 6227 CNRS, Univ de Nice Sophia Antipolis, eMail: letexier@gredeg.cnrs.fr, http://www.letexier.fr

3 PHARE - EA 3394 CNRS, Univ Paris I Panthéon Sorbonne, eMail: david@dwv.me

http://www.dwv.me.
}

61 Economie et Institutions $-\mathrm{n}^{\circ} 12 \& 13-2008-2009$ 
diverses dynamiques associées à l'innovation ouverte [open innnovation] et au développement des logiciels libres [open source]. $\mathrm{Du}$ point de vue matériel, tous ces acteurs travaillent ensemble aujourd'hui, dans tous les pays, dans une dynamique globale des programmes d'armement qui mêle liens hiérarchiques, relations de marchés, dynamiques de communautés (Amin et Cohendet 2000, 2004 ; Mérindol 2009b). Les relations y sont structurées par le pouvoir hiérarchique de l'Etat et de l'intégrateur de systèmes. Cette contribution va donc s'interroger sur les voies et moyens qui permettent d'envisager des modèles de coopération stables entre ces acteurs, et d'imaginer les politiques publiques qui permettent d'influer sur le design de ces organisations.

S'inscrivant dans la filiation de Nelson et Winter, le terme " innovation" sera utilisé au cours de cette contribution dans son sens le plus large, qui recouvre tout le processus associé à la dynamique du changement technologique portant des produits vers un usager. Globalement, cet article s'inscrit dans la logique de la synthèse SYS (Stanford - Yale - Sussex) décrite par Dosi, Llerena et Sylos Labini (2006), et reprend à son compte la référence aux propriétés communes de l'information et de la connaissance qui impactent la dynamique de l'innovation. En particulier, cette synthèse reconnaît combien les relations entre la connaissance scientifique, l'innovation technologique et leur exploitation économique sont inter-reliées et inextricables. Cette contribution va s'attacher à caractériser le statut de la connaissance et des actifs détenus par les différents acteurs, de sorte à permettre une investigation précise des liens qui permettent la coordination entre les firmes, les acteurs publics et les diverses communautés présentes. Trois domaines particuliers de la littérature scientifique seront mobilisés à cet effet : les développements sur l'intégration des systèmes (Hobday, 1998 ; Prencipe, Davies, Hobday, eds. 2003) ; les contributions sur l'innovation ouverte (Chesbrough, 2003; Chesbrough, Vanhavaerbeke, West, eds. 2008) ; les travaux sur les logiciels libres (Lerner et Tirole, 2001 ; Lerner et Tirole, 2002 ; von Hippel et von Krogh, 2003). Une des passerelles qui permettra de développer une analyse conjointe de ces domaines se trouve dans les contributions de l'économie de la connaissance qui développent la notion d'actif de connaissance (au sens de von Hippel, 1994).

La littérature relative au développement du logiciel "libre " base une grande partie de ses apports dans l'identification de règles de participation permettant de concilier les intérêts des différents acteurs participant aux activités du "libre " (Lerner et Tirole, 2001; von Hippel et von Krogh, 2003 ; von Krogh et von Hippel, 2006). L'implication de catégories d'acteurs appartenant aux sphères civiles et militaires ne modifie pas particulièrement cette démarche, car les acteurs issus des communautés du logiciel libre ne se singularisent 
pas vraiment des scientifiques associés aux autres champs de recherche mobilisés pour les programmes d'armement. Cette forme d'hybridité présente seulement quelques degrés d'originalité par rapport à la littérature sur les logiciels libres (Richardson, 1972 ; Howells, 1996; Azoulay, 2004). La calibrer de façon précise supposerait une investigation particulière en sociologie sur les motivations "politiques " des acteurs du logiciel libre, et sur leurs réticences à travailler directement ou indirectement pour la Défense. Cette contribution ne cherche pas à traiter ce point particulier.

Cette contribution va détailler les mode de coopération entre les acteurs, en analysant les liens entre acteurs marchands et nonmarchands, acteurs publics et privés, acteurs visant une logique de diffusion et une logique de privatisation / appropriation. La réalité des programmes d'armement montre que chacun de ces régimes trouve le moyen de converger avec les autres sur des modes hybrides de fonctionnement qui servent chacun des intérêts collectifs et privés. Après avoir identifié les variables majeures qui permettent de les caractériser, cette contribution va exposer pourquoi les régimes " hybrides" atteints sont stables et enclins à contribuer sous des formes pérennes à la mission régalienne de Défense et de Sécurité.

Cette contribution est structurée comme suit. La section 2 présente les faits saillants de l'opposition entre les dynamiques de l'innovation ouverte et fermée, et introduit dans ce cadre les aspects particuliers relatifs au recours à des logiciels libres. Ces développements s'inscrivant dans la cadre des activités relatives au secteur aéronautique, spatial et Défense (ASD), la section 3 retracera les aspects marquant la place des logiciels et de l'électronique dans les programmes complexes du secteur. Cette évolution induit des bouleversements dans l'adéquation entre les frontières de la division du travail et de la division de la connaissance au sein du secteur, qui justifient progressivement le recours à des logiques hybrides mêlant dynamiques de l'innovation ouverte et fermée. La section suivante présentera un programme technologique particulier, porté par l'industrie (et en particulier les intégrateurs de systèmes) et les acteurs publics pour améliorer l'économicité générale des coûts de transaction dans le secteur. Ce projet repose à la fois sur une démarche d'innovation ouverte et sur des logiciels open source. Ces réflexions conduisent ensuite à développer dans les sections suivantes des analyses plus précises du choix des acteurs (publics et privés) de "basculer" vers des solutions libres ou ouvertes. La section 5 se concentre sur l'analyse des questions relatives aux droits de propriété intellectuelle. La section 6 développe les points associés à la gouvernance des organisations dans les cadres " hybrides " mis en place par les organisations publiques et privées concernées par cette évolution. Elle esquissera aussi les contours de 
politiques publiques utiles pour orienter les stratégies d'innovation ouverte dans le secteur ASD. La section 7 conclut.

\section{L'appropriation dans la dynamique de l'innovation " ouverte"}

Longtemps assimilée à un mystérieux troisième facteur de production, l'innovation a rapidement été considérée comme un des principaux moteurs de la croissance. Historiquement, la vision entrepreneuriale s'est traditionnellement appuyée sur un modèle de développement cherchant à capitaliser les gains de la démarche innovante au sein de la firme. La disposition des entreprises à innover repose sur la capacité à préserver en leur sein la propriété et la valeur ajoutée des biens issus de la dynamique de l'innovation et, par conséquent, à raisonner sur la valorisation à partir des retours sur investissement attendus. Ce modèle d'innovation suggère que le succès d'une démarche innovante repose sur le contrôle que peuvent exercer les acteurs commerciaux sur leur production et sur leurs actifs. Le modèle d'innovation fermée repose ainsi sur une démarche de contrôle, dans laquelle les relations entretenues avec les acteurs extérieurs ne peuvent être que concurrentielles. La recherche de l'innovation tend ici à favoriser des dynamiques concurrentielles schumpetériennes, selon lesquelles le vainqueur de la course à l'innovation valorise au mieux son activité.

Ce modèle d'innovation a sans conteste donné lieu dans les années 1970 et 1980 à de formidables réussites industrielles. Elles ont été appuyées par l'établissement d'un cadre institutionnel destiné à favoriser la généralisation des pratiques d'internalisation des activités de recherche, de développement et de contrôle des canaux de distributions. Avec la prise en compte progressive des activités liées aux réseaux de connaissances (knowledge based economies), les années 1990 ont révélé des limites et nuancé les bienfaits systématiques du modèle schumpetérien: les seules ressources internes de production ne suffisent plus pour répondre aux nouveaux besoins productifs et ne parviennent plus à alimenter la démarche innovante des entreprises. Callon et al. (1999) expliquent que la connaissance est assimilable à un bien car, jusqu'à un certain point, elle ressemble à des informations. Leur valeur économique dépend alors de leur statut codifié ou incorporé, qui va alors conduire à analyser le statut de bien public (non-rivalité et non-exclusion) et leur niveau de spécificité. Les connaissances codifiées présentent un fort niveau de généralité et leur appropriation comporte un coût très élevé. En revanche, les connaissances incorporées sont rivales et leur coût d'appropriation demeure faible (Callon, 1999: 19-22). Appliquées aux actifs de connaissance, les questions de complémentarité et de concurrence reposent largement sur ces variables. Elles ouvrent sur une série de conclusion pour la

64 Economie et Institutions - n²12\&13-2008-2009 
dynamique de la R\&D: l'internalisation exclusive des activités de R\&D a non seulement montré qu'elle n'était pas en mesure de satisfaire l'ensemble des besoins innovants, mais a également souligné qu'elle pouvait mener à la production de facteurs inexploitables directement par l'entité qui la développe. Ce modèle d'innovation doit être amendé et complété.

Chesbrough (2003) définit le modèle d'innovation ouverte comme un modèle selon lequel des entités productrices exploitent non seulement leurs ressources internes mais peuvent également employer des facteurs de production proposés par des acteurs producteurs extérieurs. Le modèle d'innovation ouverte repose sur l'idée majeure qu'un acteur producteur peut utiliser des ressources internes et externes à sa firme, tout en développant en parallèle des schémas de valorisation de sa propre activité. Le modèle 'ouvert' représente par conséquent un modèle où la valorisation d'une activité ne repose pas uniquement sur la stricte exploitation de ressources internes. Au contraire, le modèle expose que les sources d'innovation peuvent être développées par d'autres acteurs, actifs matériels et de connaissance devenus accessibles par le biais de réseaux, de communautés et de mécanismes de marché. L'adoption du modèle 'ouvert' offre alors des options nouvelles pour se maintenir dans la course à l'innovation. S'ensuivent de nouvelles tendances de marché et l'introduction de nouveaux mécanismes de valorisation de l'activité productive, qui sont distincts des mécanismes entrepreneuriaux schumpetériens. Certains auteurs ont pu suggérer que la transition d'une approche purement individualiste à une approche davantage collective pouvait conduire à l'émergence d'effets d'opportunisme dont le poids peut être finalement néfaste à la juste définition d'un modèle d'allocation efficace des ressources (Hardin, 1968). Cette vision apparaît aujourd'hui très nuancée dans le cas où la nature publique des biens échangés est avérée (Foray, 2004). Dosi, Llerena et Sylos Labini (2006 : 1462) expliquent que les frontières entre $R \& D$ " ouverte " et $R \& D$ " appropriable " doivent être traitées avec pragmatisme car elles demeurent malaisées à distinguer. Ils insistent en particulier sur le fait que l'appropriation des connaissances est justifiée si et seulement si elle se trouve associée à des incitations en faveur de l'innovation. Comme ils l'expriment, les incidences du statut public ou privé des connaissances ne sont pas généralisables, car les dynamiques sectorielles et les paradigmes technologiques prévalent largement pour comprendre les incitations en faveur de la création de nouvelles connaissances à partir d'un cadre public ou privé. A l'image du développement des sciences, une approche fondant la dynamique de l'innovation sur la coopération semble en mesure de fournir des résultats intéressants, potentiellement plus satisfaisants au regard de l'économie du bienêtre que ceux atteints dans la vision traditionnelle puisque la 
diffusion des actifs productifs permet d'atteindre une meilleure diffusion des actifs de production (Merton, 1973 ; David, 2004).

L'ouverture des activités de production et le développement de l'accessibilité aux actifs de production représentent un nouveau paradigme pour la dynamique de l'innovation. Cette ouverture revient non seulement à associer les activités de production d'entreprises autrefois perçues comme de simples concurrentes, mais également à intégrer celles d'organisations qui ne sont pas mues par des intérêts strictement marchands. Depuis le développement des technologies de l'information et de la communication, notamment de l'Internet, de nouvelles organisations productives de connaissances se sont constituées sous la forme de communautés. En comparaison avec les organisations de production de type marchand, les communautés rassemblent des acteurs motivés par un besoin d'utilisation précis pour un bien qu'ils tiennent à créer via la communauté (Wenger, 1998 ; Cohendet et al., 2001). Véritables 'prosommateurs' (Toffler, 1980), certains utilisateurs manifestent des aptitudes à l'innovation et au développement technologique qui les sortent de la position de simple consommateur. L'activité de production n'est donc plus désormais uniquement assurée par les acteurs marchands traditionnels, car de nouveaux acteurs non-marchands - lead users - contribuent directement à la dynamique marchande de l'innovation (von Hippel, 1986 ; von Hippel, 1988 ; von Hippel 2005). La particularité de ce modèle de développement technologique ouvert, ancré dans les communautés, favorise le développement de nouvelles logiques de production qui tirent bénéfice de l'expression créative des participants aux communautés. Ainsi, ces organisations alternatives peuvent proposer des solutions exploitables par d'autres organisations tout en échappant au modèle d'innovation schumpetérien.

Basculer du paradigme d'innovation fermée vers celui d'innovation ouverte requiert toutefois l'instauration de mécanismes d'ajustement mutuels qui confortent la démarche coopérative et évitent la généralisation de comportements opportunistes. Que ce soit dans le cadre d'une coopération interentreprises ou dans le contexte d'une coopération entre une entité marchande et une organisation non-marchande, des règles de coordination doivent être identifiées pour que les attentes des acteurs marchands associées à la logique de l'appropriation soient compatibles avec celles d'utilisateurs-producteurs, qui relèvent d'une démarche plus collective et communautaire. En soi, un modèle 'privé-collectif' d'innovation doit alors être défini pour que la démarche d'innovation 'ouverte' soit fructueuse (von Hippel et von Krogh, 2003). Le succès s'appuie sur la conciliation des activités et des intérêts individuels des différentes parties prenantes (stakeholders). Ce constat suggère 
l'identification des mécanismes d'ajustement qui ciblent des processus d'innovation impossibles à concrétiser dans le modèle d'innovation fermée. L'identification de tels mécanismes conduit ensuite à la définition de modèles hybrides de production, pour lesquels des règles de coopération sont établies pour permettre de concilier en même temps les intérêts privés de la firme et les intérêts communautaires. Définir de tels cadres n'est pas chose aisée, mais cette étape est incontournable pour tirer parti du modèle d'innovation 'ouverte'. La capacité d'une organisation à apprécier les caractéristiques du modèle d'innovation 'ouverte' découlent donc de l'hybridité retenue pour les relations avec les acteurs communautaires, qui à son tour conditionne l'efficacité (sociale) du modèle (Chesbrough et Rosenbloom, 2002 ; Chesbrough, 2003). La nature des liens dessinés entre acteurs conditionne la pertinence du changement de paradigme et caractérise l'ensemble des capacités développées concrètement par ces acteurs innovants pour atteindre une situation mutuellement profitable (Lorenzoni et Lipparini, 1999; Dahlander, 2007 ; Dahlander et Magnusson, 2008 ; Flowers, 2008).

L'emploi croissant du modèle d'innovation ouverte, à l'image d'IBM Corporation, révèle en théorie des potentialités de gains plus élevées que celles atteignables dans le modèle 'fermé'. Toutefois, la nature des bienfaits perçus dépend des stratégies de positionnement mises en place par les entités basculantes pour tirer parti du modèle 'ouvert'. Les caractéristiques du modèle hybride de production sélectionné, et indirectement le montant des efforts de coordination requis pour que des acteurs coopérants dégagent mutuellement des gains, participent par conséquent à la modification des stratégies industrielles prévalant dans le domaine (Teece, 1986; Howells, 1996). Jacobides, Knudsen et Augier (2006) expliquent que les architectures industrielles sont la source de la structuration de la division du travail pour un secteur particulier. Elles comprennent à la fois les aspects institutionnels, techniques et physiques, et procurent un cadre aux transactions et aux interfaces entre les acteurs. Toute la dynamique des coûts de transaction s'ensuit alors, pour expliquer la logique de dépendance (ou d'interdépendance) entre les contributions des acteurs et les actifs de connaissance qui caractérisent leurs rôles et leurs contributions à la chaîne de création de valeur. Il devient alors possible d'identifier la complémentarité des facteurs et leur mobilité comme deux composants de la co-spécialisation, ou de ce que Teece désignait comme une forme de dépendance bilatérale. La complémentarité influence les combinaisons de facteurs et de connaissances qui affectent le niveau de valeur qui sera crée (en fonction du " fit " entre les contributions) ; la mobilité impacte en revanche le pouvoir de négociation des acteurs, et donc la part de valeur à laquelle ils ont accès. Il devient alors possible d'entrer dans l'analyse de la 
dynamique de l'innovation ouverte sur la base des nuances apportées par cette lecture ( $c f$ West et Gallagher, 2006: 87). Sur quelles bases les acteurs entrent-ils dans ce type d'interaction ? En quoi le secteur de la Défense et de la Sécurité procure-t-il ici un cadre d'investigation original pour la prise en compte des politiques publiques fondées sur l'innovation ouverte? Comment les engagements de ressources en faveur de cette dynamique peuvent-ils intégrer la concurrence entre les firmes dans le même temps que la prise en compte de la dynamique des communautés ? Ces éléments doivent en ouvre conserver une cohérence avec les caractéristiques de gestion des droits de propriété intellectuelle dans ce secteur.

La dynamique des logiciels libres illustre parfaitement la question de l'innovation ouverte. Elle contraste avec le modèle industriel fondé sur des logiciels propriétaires sur la base de trois éléments développés dans la littérature scientifique : la philosophie associée aux droits de propriété intellectuelle, la production dans un cadre collaboratif, la présence de firmes aux stades de la R\&D sur les logiciels open source et de l'industrialisation de projets qui mobilisent ces développements. West et Gallagher (2006 : 89) expliquent que les logiciels libres représentent une manifestation de linnovation ouverte, singularisée par trois points saillants. Le premier porte sur les droits d'usage de la technologie; le deuxième renvoie à la nécessité de poursuivre des formes de développement collaboratif pour conserver les avantages associés à cette forme d'interaction; le troisième concerne le retour sur investissement associé à l'entrée des firmes dans cette dynamique, qui peut d'autant plus se révéler en contradiction avec la vie des communautés que les usages attendus concernent des programmes de Sécurité et de Défense. L'enjeu est important. Comme l'indiquent Amin et Cohendet (2004), cette démarche demeure malaisée car elle repose sur des comportements antinomiques : la gestion des droits de propriété intellectuelle renvoie à la position d'un inventeur qui se protège contre le monde extérieur dans le contexte "propriétaire ", alors que l'enjeu fondamental repose sur une logique de conviction, de persuasion et d'échange dans le modèle "ouvert". Les schémas d'interaction et d'incitation pris en compte par les politiques publiques en sont totalement bouleversés.

\section{Les questions relatives au software dans les programmes de Défense}

Dussauge et Cornu décrivent (1998: 14) l'envolée de la part de l'électronique dans les programmes militaires. Ils évoquent des chiffres ciblés sur les chars de combat français, qui passent de $10 \%$ pour le char AMX 30 mis en service à la fin des années 1960 pour atteindre plus de $50 \%$ dès les systèmes comme le char Leclerc qui ont été développés dans les années 1980. Compte tenu du progrès 
technique, ces montants se maintiennent entre $50 \%$ et $60 \%$ du coût total d'acquisition et de maintenance des plateformes militaires majeures (avion de combat, char de combat, sous-marin ou porteavion). Les analyses des programmes actuels (Dombrowski et al, 2002 ; Versailles 2005) exposent que ces coûts peuvent aller au-delà de ces normes pour les systèmes de commandement caractérisés par des fonctions de communication et de cryptage qui sont le cœur de la guerre en réseaux et de la révolution des affaires militaires. Pour leur part, les coûts de développement associés aux logiciels ont littéralement explosé au niveau des plateformes majeures depuis que l'électronique gère à la fois les plateformes (par exemple via les commandes de vol électriques pour les avions d'armes) et les systèmes de mission (tous les éléments relatifs à la fusion des données tactiques et à la gestion des transmissions). Le développement des programmes par "blocs " ou " standards " rend compte d'ailleurs très largement de l'implémentation progressive des fonctionnalités liées aux logiciels, et des bouleversements induits par l'introduction de la nouvelle version d'un module (ou sous-module) particulier dans le système complet. Personne ne sait quantifier cette évolution de façon précise du point de vue budgétaire, sauf à exposer que les technologies de l'information sont devenue cruciales et que le plan de charge associé au développement des logiciels s'accroît inexorablement. L'effet système découle d'une très forte imbrication entre les couches logicielles présentes dans le développement de chaque génération de module et sous-module, dans l'interaction des modules au niveau des plateformes, dans l'interaction des plateformes entre elles au sein du réseau de commandement, et dans les organes spécifiques au réseau de commandement lui-même. Toute modification ou mise à jour doit maintenir le système cohérent et préserver la compatibilité entre des matériels par nature hétérogènes. Les couches logicielles liées aux programmes d'armement représentent donc la cause et la conséquence d'une démarche d'amélioration incrémentale permanente, mais aussi de coûts de développement qui se cumulent dans des effets de spirale [spiralling development costs]. Cela se traduit par des budgets de l'ordre de 14 G\$ (courants) en 2006 pour les coûts de développement informatique associés aux programmes militaires américains quand, en 2003, $8 \mathrm{G} \$ 4$ portaient seulement sur des adaptations des lignes de code informatique requises pour la finalisation des programmes ou la suppression des problèmes liés au code dans les programmes livrés aux forces. De façon générale, la moitié des programmes d'armement américains s'achève avec un dépassement du volume de

4 Soit juste le montant requis pour acquérir deux porte-avions nucléaires de 100000 tonnes (classe CVN 21).

69 Economie et Institutions - n²12\&13-2008-2009 
code informatique d'au moins $25 \%$ par rapport aux prévisions (Le Texier et Versailles, 2009:20).

Cette situation conduit à renforcer la fonction d'intégration, incontournable dans le secteur de l'armement (Sapolsky, 2003). La fonction repose sur le rôle de la firme intégrateur de systèmes, qui joue un rôle central dans la coordination du travail et dans la conception des systèmes complexes, à l'intersection des relations entre les équipementiers, les autres fournisseurs et l'usager final des systèmes de Défense. La frontière des connaissances entre les firmes évolue avec le temps, mais elle découle directement de la complexité des programmes et de la nature des interfaces qui relient les systèmes entre eux. Les acteurs spécifiques aux technologies de l'information et au développement des logiciels montent donc de façon automatique dans la chaîne de la valeur ajoutée. La division du travail est particulièrement marquée dans le secteur de l'armement, à cause de la multiplicité des modules pris en compte dans la création de systèmes complexes que représentent les plateformes de combat et les systèmes de commandement en réseau. La variété des composants et sous-composants a augmenté de façon très importante avec le temps et le progrès technique, conduisant à des effets systémiques qui imposent de nouveaux modes de gestion de programmes d'armement: ceux-ci doivent être perçus dans leur dimension système, voire au niveau des systèmes de systèmes, car les interactions entre l'ensemble des contraintes associées à chaque module conduisent à des niveaux de complexité impossibles à imaginer dans les systèmes modulaires.

La division du travail repose sur une spécialisation des firmes autour de modules spécifiques, et par l'interaction entre organisations aux activités faiblement couplées ([loosely coupled], Brusoni et al, 2001). Tous les acteurs ont besoin d'une compétence liée aux logiciels. Dans le monde des "vieilles " technologies, les interfaces entre modules reposaient le plus souvent sur des mécanismes physiques ; aujourd'hui, le progrès technique conduit à mobiliser les technologies de l'information à tous les niveaux de ces interactions. Comme pour tous les systèmes complexes [CoPS] (Hobday, 1998 ; O’Sullivan, 2006), les structures de coordination des programmes ont pris une importance critique avec le temps pour endosser la responsabilité des décisions techniques et de l'analyse des arbitrages coûts-sophistication-qualité pour les programmes. C'est à ce seul niveau de décision, et sur la seule base de ces arbitrages, que le coût total de possession peut se trouver plafonné. L'intégrateur du système (ou du système de systèmes) doit donc se reposer sur des compétences multi-technologiques et organisationnelles, qui sont supposées lui procurer une vue d'ensemble permettant les arbitrages et les modalités du travail au sein du réseau. Parmi les arbitrages cruciaux figurent toutes les 
décisions de management de l'innovation et de répartition du travail (in-sourcing, out-sourcing, joint-sourcing), qui ont un impact direct sur la gestion de la fiabilité du système ( $\mathrm{y}$ compris sa supériorité opérationnelle future), des délais de production (par le biais de l'analyse de la maturité des technologies) et de coûts. Cette situation est d'autant plus délicate à gérer que la finalisation du programme repose sur la mise en œuvre de couches logicielles dont le développement accompagne les tests et essais tout au long du programme, dans le rythme de développement par "blocs", requérant même une mise au point in situ dans la logique même des opérations pour aller au bout de la fiabilisation du système (de systèmes).

Chaque groupe industriel s'est donc doté des compétences d'intégration requises pour asseoir sa position. Les acteurs majeurs se caractérisent par une stratégie propre au niveau des réseaux qu'il gère, et qui non seulement le distinguent dans sa relation de pouvoir au sein du réseau d'intégration, mais singularisent aussi sa relation avec son client final de référence. Dans le cas des programmes liés à des exportations, la stratégie induite par cette position s'impose ensuite aux autres clients. La frontière entre division du travail et division des connaissances évolue donc progressivement au fur et à mesure de l'évolution des technologies (Brusoni et al., 2001 ; Brusoni et Prencipe, 2006 ; Versailles et Mérindol, 2009). Pour chaque famille particulière de système complexe, il est possible de spécifier des architectures industrielles spécifiques qui rendent compte des dynamiques de production et d'innovation spécifiques aux acteurs présents. Tout renvoie à la prise en compte des dépendances bilatérales entre les contributeurs au réseau d'intégration, ce qui découle à la fois de la contribution des firmes à la création de valeur ajoutée pour les produits et de la préparation des programmes futurs. Il convient alors de s'interroger sur la convergence entre les deux modèles servant de références à cette contribution : d'une part le modèle d'intégration de systèmes et, d'autre part, le modèle de l'innovation ouverte. Cette recherche repose pour une grande partie sur le constat d'un dépassement de la situation qui avait prévalu jusqu'au début des années 1990, et qui était caractérisée par une logique d'appropriation et de croissance externe gérée par les intégrateurs de systèmes. Ceux-ci faisaient entrer les innovations requises par les programmes à développer, principalement sous la forme de croissance externe de l'un ou l'autre des contributeurs au réseau d'intégration ou de l'accès d'une PME à un statut de soustraitant de spécialité. La saine gestion du réseau d'intégration conduisait ensuite à partager l'accès aux nouvelles ressources parmi les partenaires stables du réseau. Cette situation ne correspond plus aux interactions actuelles régissant l'innovation, qui renvoient bien plus à la coexistence entre divers acteurs - publics, privés et

71 Economie et Institutions $-\mathrm{n}^{\circ} 12 \& 13-2008-2009$ 
marchands, privés mais non marchands, communautés, réseaux informels.

Cette nouvelle situation ne signifie pas pour autant que les programmes d'armement se sont ouverts à un régime d'innovation ouverte. La réalité se situe plutôt face à des stratégies plus complexes. Plusieurs exemples récents permettent de confronter ces situations hybrides aux concepts décrits dans cette communication. Ainsi, pour le programme Future combat system, composé de dix-sept programmes relatifs à des plateformes destinées à l'US Army, Boeing agit comme intégrateur de systèmes de systèmes, mais se repose sur les compétences de SAIC pour ce qui concerne les spécificités de développement et de mise au point des diverses couches de logiciels. SAIC y a même gagné une position d'intégrateur. Lockheed Martin illustre une stratégie différente où le développement des couches logicielles relatives à l'intégration des systèmes (et a fortiori des systèmes de systèmes) demeure internalisée [in-sourced] pour des raisons stratégiques, ce qui conduit ce groupe industriel à produire, à lui tout seul, chaque année, plus de lignes de codes que tous les acteurs américains du secteur des technologies de l'information réunis, soit IBM, Microsoft et Intel inclus (Herz et al., 2006). En France, pour les avions d'armes et les drones de combat mais pas au niveau des systèmes de systèmes puisqu'elle n'y est pas présente, une firme comme Dassault illustre une stratégie basée sur la prise en compte de contraintes différentes (Versailles, 2005 ; Versailles et Mérindol, 2009). Sa stratégie d'intégrateur de systèmes tend à ressembler à celle de Lockheed Martin, y compris dans le choix d'inventer des outils informatiques de développement 3D spécifiques (la suite logicielle CATIA) et des langages informatiques particuliers adaptés au hardware informatique maison qu'ils mettent en œuvre pour permettre des traitements "temps réel". En revanche, les logiques de normalisation mises en œuvre par l'OTAN imposent souvent d'abandonner des solutions technologiques pour adapter les standards qui découlent du pouvoir de marché conjoint du département de la Défense et des industriels américains.

\section{Une plateforme développée sur la base de l'innovation ouverte : Certipath et la certification des échanges}

Travaillant sur la relation entre innovation ouverte et logiciels libres, West et Gallagher (2006 : 100-2) explicitent que la référence à la dynamique de l'innovation ouverte nécessite de faire un lien avec un modèle d'affaires particulier au niveau d'une entreprise, de sorte à capturer de la valeur et à justifier d'un retour sur investissement. Ils mentionnent que de nombreux projets open source sont gérés sans motivation pécuniaire, ce qui reflète tout à la fois des situations où le sponsor n'a pas réussi à créer un modèle d'affaires viable et 
d'autres cas où il l'a, au contraire, abandonné au grand public car il ne pouvait plus le nourrir. Les auteurs énumèrent divers exemples pour illustrer ces configurations. Dans la Défense, le dernier exemple est plus rare. Il peut être illustré par Boeing qui a favorisé et soutenu le développement d'un système de prototypage dénommé Helda proxy. Ayant décidé de ne pas poursuivre le développement de cette compétence et de cette solution dans un but commercial, Boeing a abandonné cette suite logicielle aux communautés du libre pour lui laisser une forme de vie autonome.

Cette section va détailler l'analyse d'un cas particulier utile à l'analyse de l'interaction entre le modèle ouvert (développement logiciel libre ou innovation ouverte) et le pouvoir structurant (pouvoir de marché, pouvoir de décision, pouvoir d'arbitrage) d'un intégrateur de systèmes. Certipath ${ }^{5}$ est un consortium mis en place dans le but de renforcer et de simplifier le management de la confiance entre les acteurs des programmes de l'aéronautique, du spatial et de la Défense (ASD). Les services proposés couvrent les modalités de sécurisation des échanges, les règles d'échange et les modalités de recours aux interfaces entre acteurs d'un projet technologique ou d'un programme, les niveaux de sécurisation et de d'identification, les règles de restriction d'utilisation. Les interfaces gérées par Certipath et TSCP reposent sur les outils usuels de l'internet : email, service de transfert de fichiers, forums. Ce service commercial procure une infrastructure de gestion des interactions entre les acteurs des firmes contribuant aux projets technologiques $(R \& D$, production) à partir d'un système d'authentification fondé sur des clés publiques (PKI, public key infrastructure). Pour prendre la mesure de cet enjeu, il convient de garder à l'esprit que plus de deux cent mille firmes différentes se connectent aux différentes bases de données du Pentagone pour contribuer à ces programmes dans le cadre des appels d'offres, puis des phases de R\&D et de production. Les connexions concernent plus de trente mille entreprises différentes chaque jour, à partir d'une multiplicité d'individus en leur sein. La technologie retenue combine des séquences d'authentification et de cryptologie qui relèvent au départ d'une technologie open source. Le service proposé repose donc sur l'appropriation de solutions technologiques libres au profit d'une mission particulière qui se trouve dans l'intérêt conjoint (et identique) de tous les membres du consortium. Le service peut être commercial, ce qui est le cas avec Certipath. L'originalité de

5 Cf www.certipath.com, www.tscp.com; cette analyse est élaborée pour partie sur la base des entretiens et présentations qui se sont déroulés pendant le workshop FLOSS 1.5 organisé par les auteurs à Salon de Provence en avril 2007. Les commentaires développés ici n'engagent que les seuls auteurs.

73 Economie et Institutions - n²12\&13-2008-2009 
Certipath et TSCP réside dans l'échelle du service: la démarche dépasse le niveau d'une seule entreprise ou d'un seul projet pour procurer un outil qui permet de vérifier les droits d'accès de tous les contributeurs aux divers réseaux du secteur aéronautique, spatial et Défense, dans un cadre international adapté à la réalité des programmes internationaux. En résulte un programme de certification dénommé TSCP, acronyme qui signifie "Transglobal Secure Collaboration Program".

Le consortium Certipath regroupe les principaux industriels intégrateurs de systèmes américains et européens (EADS/Airbus, Northrop, Boeing, Rolls Royce, Raytheon, Lockheed Martin, BAe Systems, Finmeccanica), ainsi que trois maitres d'ouvrage (le Département de la Défense américain, les ministères britannique et néerlandais de la Défense). L'utilisation de ces services est réalisée au cas par cas sur la base de décisions prises par les intégrateurs de systèmes et par les deux maîtres d'ouvrage, lesquels ont ensemble conçu, orienté et évalué les contenus de TSCP et Certipath à partir des années 2005-2006. EADS a été la première entreprise européenne certifiée par Certipath, moins de deux ans après Lockheed Martin (première firme entrée dans ce système). La coordination se trouve donc gérée à partir de modalités hiérarchiques, où le haut de la pyramide de l'intégration des systèmes détient toutes les modalités de contrôle et de décision associées à la définition puis à l'implémentation des interfaces de coordination. A l'intérieur de chacune des organisations, les acteurs doivent se conformer à ces règles d'échange et adapter en conséquence leurs comportements. Si les solutions proposées sont considérées comme très performantes du point de vue de la sécurité des échanges, il n'en demeure pas moins que leur usage (comme toujours en pareil cas) et les normes qu'il impose de façon automatique sont jugées contraignants et peu conviviaux.

Les services de Certipath et TSCP sont gérés conjointement par trois structures : Exostar (spécialiste de la gestion des relations au sein de la supply chain pour le secteur ASD aux Etats-Unis); Arinc (le responsable américain de la mise en œuvre des réseaux de communication air-sol pour l'aéronautique civile et militaire, positionné aussi sur les technologies de la guerre en réseau dans le cadre du network centric warfare) ; SITA (le spécialiste européen des infrastructures de communication pour le transport aérien civil, en charge des communications sécurisées par protocole internet pour $90 \%$ des compagnies aériennes mondiales). Les entretiens réalisés autour de Certipath ont d'ailleurs permis de souligner que la Commission européenne a explicitement soutenu l'engagement des firmes européennes dans ces démarches, y compris par des financements associés au 6ème Programme cadre de recherche et de développement (PCRD, FP6). Le modèle porté par Certipath et TSCP

74 Economie et Institutions - n²12\&13-2008-2009 
favorise l'accès des firmes européennes aux différents marchés et contribue à l'efficacité globale de la base industrielle européenne dans le secteur. Le premier nom de TSCP manifestait d'ailleurs clairement la volonté d'accéder aux réseaux d'intégration dirigés par des firmes américaines, puisque " $\mathrm{T}$ " signifiait alors " transatlantic" et non pas " transglobal".

Le programme répond à plusieurs principes fondamentaux associés à l'innovation ouverte (Chesbrough, 2003: xxvi). Le processus d'innovation résulte de la mobilisation de réseaux de recherche intéressés à l'amélioration globale du fonctionnement du secteur aéronautique, mais qui ne sont pas directement associés à la chaîne de valeur d'une entreprise spécifique. Certipath et les services dérivés de TSCP impactent directement le fonctionnement du secteur en tant que système d'interaction. Les bénéficiaires du service qui sont parties prenantes au consortium bénéficient du service innovant par le biais de leur participation, sans avoir à développer cette recherche en interne. Ils créent de la valeur pour eux-mêmes et pour le consortium sur la base de leur contribution comme lead users au réseau de développement. D'évidence, les first movers (et en particulier les plus forts des intégrateurs mentionnés plus haut) structurent les développements, mais leur influence est tempérée par les mécanismes de concertation et de coordination et par les missions de service public particulières aux trois entreprises porteurs du projet Certipath. Une seule d'entre-elles (Arinc) se trouve d'ailleurs engagée dans une logique commerciale motivée par des profits. Au total, les first movers se trouvent donc dans une situation où ils donnent l'exemple afin d'entraîner derrière eux les acteurs présents dans les réseaux d'intégration placés sous leurs responsabilités respectives, débloquant le problème de l'adhésion des autres acteurs par cet engagement (ici à la fois par l'effet de réputation et par la définition du standard). Au sein du secteur ASD, les services délivrés par Certipath et TSCP portent bien plus la marque d'une meilleure économicité des business models de chacun des acteurs du secteur qu'une amélioration des marges de l'une ou l'autre des entreprises. C'est une baisse générale des coûts de transaction au sein du secteur ASD sur le plan international que ce consortium procure, à travers un langage qui permet de gérer la confiance en temps réel sur la base d'une grammaire et d'un système de bases de données. Le gain de chaque acteur du système repose donc sur la dynamique d'acceptation de ce langage, procurant ainsi un surplus maximum aux acteurs du secteur lorsque chacun se coordonne de façon spontanée à ce nouvel intermédiaire général des échanges. Dans le processus d'innovation, le gain de chacun sera également associé à la transparence des outils mobilisés pour les échanges. Les services procurés par Certipath et TSCP se trouvent en réalité reliés directement à l'économie générale des programmes, à

75 Economie et Institutions - n²12\&13-2008-2009 
travers les perspectives de réduction des coûts de coordination et d'information entre acteurs. Le service procuré sera d'autant plus efficace que les participants à l'amélioration du système seront toujours plus nombreux, généralisant le comportement des lead users engagés initialement en faveur de l'élaboration du système en vue d'une amélioration constante de la fiabilité et de la réactivité du système de certification. Y sera nécessaire une forme de partage des droits de propriété intellectuelle sur les outils permettant à Certipath et TSCP de servir de base aux relations de confiance, qui se limitera à traduire dans la réalité les questions organisationnelles associées à la gestion des relations au sein des réseaux d'intégration. C'est ici un point majeur de l'efficacité des programmes complexes. Puisque chaque intégrateur gère les relations avec son réseau sur la base d'un modèle stratégique propre, leur présence dans les phases initiales du développement de Certipath devient donc facile à expliquer.

\section{5. "Basculement" vers l'innovation ouverte et droits de propriété intellectuelle}

Les systèmes complexes (CoPS, complex products and systems) représentent le cadre de ce développement, qui se caractérise tout à la fois par la multiplicité des acteurs, par la complexité du programme où se trouvent mobilisées de très nombreuses technologies, et par la nécessité d'apporter progressivement des mises à jour pour chacun des composants logiciels ou technologiques ( $c f$ Versailles et al. 2003; Mérindol et Versailles, 2007). Cette interaction s'inscrit dans un cadre particulier, où la Défense est représentée à la fois par des acteurs en charge de la gouvernance (par exemple au niveau des bureaux en charge des programmes ou de la politique d'acquisition), de la $R \& D$ (avec en particulier les laboratoires de recherche militaires qui dépendent du DoD et interagissent avec les structures miroir de l'industrie ou du monde académique), et par les usagers (qui sont nécessairement impliqués dans la mise au point sur le terrain et dans le développement des systèmes complexes). Contribuent aussi à cette interaction les bureaux d'étude et les centres de recherche liés à l'industrie, qui sera ensuite responsable de la production des artefacts technologiques utilisés dans les programmes (au niveau des modules ou des systèmes). Enfin, il convient d'ajouter à cette énumération les acteurs de la recherche académique qui apportent leur pierre à l'édifice par le biais de contrats avec le gouvernement ou l'industrie. Cette multiplicité d'interfaces facilite de toute évidence les échanges destinés à la finalisation des programmes d'armement, qui ne peuvent aboutir sans la mobilisation de tous ces acteurs. En revanche, elle accroît de façon exponentielle les difficultés associées à

76 Economie et Institutions - n $12 \& 13-2008-2009$ 
la gestion des droits de propriété intellectuelle car tous les acteurs cités ont accès à des informations qui, d'ordinaire, relèvent de droits particuliers et d'une protection spécifique.

Il convient de noter ici que la difficulté à gérer ces dimensions s'inscrit dans l'approche système qui a déjà été largement développée dans la lignée de Hobday (1998). Comme le développe Pavitt (2003 :85), la particularité de cette situation repose sur le fait que la modularité des programmes n'a rien à voir avec la multiplication de briques technologiques comme on pourrait l'envisager sur la base d'un jeu de Lego; les effets systémiques émergent à partir d'une multiplicité de ricochets et de valorisations croisées où les contributions des uns et des autres ne prennent sens que dans le cadre des interactions au niveau du système. L'hétérogénéité des acteurs, la maturité des technologies, les calendriers de développement (phase aval de la R\&D) sont autant de points particuliers pris en compte par les outils managériaux spécifiques à cet environnement, où arbitrages et optimisation doivent prendre en compte les dynamiques croisées et donc intervenir au niveau du système seulement (Hobday et al., 2004). Le niveau du coût de production, la nature même des investissements et la prévalence de solutions technologiques innovantes dans le système conduisent à associer toute décision à des coûts irrécouvrables et à des retards de gestion du programme propre à remettre en cause son économie ${ }^{6}$ tout entière ou les calendriers d'équipement du client.

La question-clé renvoie à nouveau à l'identification de la structure organisationnelle adéquate pour prendre en charge les diverses composantes de la gouvernance de ces niveaux de complexité. Il convient de relever que la coordination des actions s'effectue par le projet, et de souligner que la gouvernance des relations entre contributeurs à de tels programmes ne prend pas sa source dans des relations marchandes dictées simplement par la négociation de prix et de volumes de biens. Les échanges entre acteurs économiques pour de tels programmes s'inscrivent dans la durée, dans la confiance et dans des stratégies de coopération industrielle (au niveau de la $R \& D$ et de la production) qui couvrent

6 Un très bon exemple se trouve aujourd'hui associé à la gestion du programme A400M par Airbus Military. L'analyse des coûts était associée à la valorisation de solutions technologiques duales, communes en grande partie avec les avions de la gamme civile, ce qui avait conduit à définir une commande multinationale sur la base d'un contrat à prix fixe. Les soucis de développement des moteurs (turbopropulseurs à hélice) entraînent un retard de plusieurs années qui remet en cause l'équipement des clients en matière de transport stratégique, situation qui va créer de fait une rupture de capacité militaire. Si cette situation n'est pas gérable du point de vue militaire, l'issue peut être fatale au programme et à l'entreprise, puisque les clients se tourneraient alors vers l'autre industriel du duopole.

77 Economie et Institutions - n¹2\&13-2008-2009 
explicitement une succession de programmes, lesquels s'étendent individuellement sur plusieurs dizaines d'années. Rares sont les retours sur investissement qui peuvent se satisfaire d'une activité associée à un seul programme, sauf à imaginer des particularismes associées à des technologies duales et à des spin-offs strictement civils qui deviennent plus rentables que les programmes militaires. Les relations entre acteurs s'inscrivent dans un temps long. Cette question renvoie directement à la juste définition des règles de contrôle au sein d'un programme de développement, et de l'allocation d'un niveau d'autorité distinct à chacun des participants en fonction de ses compétences initiales (Henderson et Clark, 1990). L'analyse des droits de propriété intellectuelle dans l'environnement de l'innovation ouverte repose précisément sur la capacité à ouvrir leur accès pour améliorer les modèles d'affaires, dans un double sens : vers l'extérieur et vers l'intérieur de la firme.

Dans le cas d'un projet de développement technologique mobilisant les logiciels libres, la discussion sur les droits de propriété intellectuelle ouvre sur les développements traditionnels en matière de licences et de brevets. Pour l'essentiel, les programmes d'armement mobilisent des lignes de codes et des langages. Ils ne font appel à des logiciels finalisés que de façon exceptionnelle. C'est dans cette perspective qu'il convient de faire le lien avec la littérature scientifique pour analyser les aspects marchands (CasadesusMasanell et Ghemawat, 2006 ; Economides et Katsamakas, 2006) et organisationnels (Muffatto, 2004 ; Dahlander, 2007). Les auteurs s'accordent pour souligner le rôle essentiel des modèles de licence dans le dessin des trajectoires concurrentielles et dans le succès ou l'échec d'une exploitation commerciale du modèle "libre". La singularité de ce développement en matière de droits de propriété intellectuelle porte précisément sur le fait que la relation entre le développeur et le client ne se fait pas directement. La contribution du logiciel libre est noyée dans le développement du système de Défense, et participe directement à son efficacité sans que l'output "logiciel libre " ne puisse être appréhendé de façon directe par le client. Au niveau de l'usager final qui pilote un avion de chasse en conditions de guerre ou qui utilise un système de commandement et de ciblage en étant pris sous le feu de l'ennemi, peu importe que le logiciel soit libre ou pas. L'important est que le système fonctionne. L'efficacité de la contribution du développeur de logiciel libre n'est appréciée que par le bureau qui gère le programme et par les autres contributeurs au développement et à la fabrication du programme, donc en premier lieu par l'intégrateur du système et par les industriels concernés par les interfaces avec le code développé.

Le recours croissant aux solutions logicielles issues du mode de production 'open source' à des fins marchandes représente sans nul doute un exemple de basculement du paradigme d'innovation 
fermée vers celui d'innovation ouverte (Chesbrough, 2003). Aux Etats-Unis, le Pentagone n'a jamais été enclin à privilégier particulièrement les acteurs du logiciel libre au détriment des firmes spécialisées dans le logiciel propriétaire. Ces dernières ont toujours démontré une forte proximité avec le DoD, et construit une partie de leurs compétences sur la base de programmes militaires. Ainsi de Microsoft qui, dans un premier temps, a développé son environnement Windows NT à partir de commandes de l'US Navy qui recherchait un système sécurisé pour gérer le réseau constitué de ses plateformes. Dans les années 1990, l'ouverture à d'autres acteurs et à d'autres OS (operating system) prend sa source dans le refus de Microsoft de contribuer aux nouveaux programmes de porteavion nucléaire (à l'occasion du développement du CVN 77) et de frégate (programme $\mathrm{DD}(\mathrm{X})$ initié par le DD-21). Dombrowski et al. (2002 : 27) exposent que Microsoft a privilégié le marché civil où la rentabilité des investissements était plus grande: d'une part cette firme n'a pas cherché à adapter son produit grand public aux besoins spécifiques de l'US Navy, et d'autre part sa contribution au programme est demeurée largement "virtuelle " (sic). Microsoft illustre parfaitement la situation d'un basculement réalisé au détriment de la Défense.

La motivation des acteurs qui basculent en faveur des programmes de Défense repose sur une stratégie symétrique. Les programmes de Défense apportent des budgets et du plan de charge. Ils permettent d'acquérir des compétences particulières. Ils permettent de participer à des réseaux d'acteurs qui ouvrent la porte à de nouvelles applications et à de nouvelles valorisations. Dans le cas de producteurs basculants, le rôle des droits de propriété intellectuelle semble essentiel. Leur représentation - sous forme de brevets - s'accorde avec les activités de recherche et développement (Guesnerie et Tirole, 1985) et l'évolution de leur emploi tient compte du changement d'implication - intégrée ou coopérative - des acteurs développeurs au sein des réseaux associés aux programmes d'armement. Ainsi, contrairement au paradigme d'innovation fermée pour lequel l'expression d'idées se justifie exclusivement par la monétarisation qu'elle engendre, le paradigme d'innovation ouverte suggère que la poursuite d'une démarche innovante doit s'appuyer sur un marché de droits de propriété intellectuelle et ainsi un jeu d'échanges de brevets avec des acteurs extérieurs (Chesbrough, 2003 ; Graham et Mowery, 2008; Simcoe, 2008) conformément à une règle marchande. En tant que telle, ce n'est pas l'estimation de la valeur des actifs qui prime lors de la mise en évidence des droits de propriété intellectuelle au sein du réseau. La frontière se situe bien plus vers l'extérieur du réseau d'intégration qu'au niveau de la firme elle-même : la poursuite de la dynamique de l'innovation au sein du réseau d'intégration et dans le cadre du programme 
d'armement induit que les connaissances issues de ces interactions sont par nature plus poussées que ce qui se trouve codifié au sein du brevet. Cet enregistrement peut alors revêtir la forme d'une protection contre les acteurs industriels extérieurs au réseau, qui servirait tout à la fois la stratégie privée de la firme qui dépose le brevet et la stratégie collective du réseau d'intégration. A ce stade, la démarche conduisant au brevet comporte une dimension stratégique pour la reconnaissance de la firme déposant le brevet envers la communauté que représente le réseau d'intégration. Ce résultat traduit le point souligné par Amin et Cohendet (2004), et présente le brevet comme un vecteur du signalement de la compétence dans le réseau bien plus que comme moyen d'une monétarisation de sa valeur dans l'échange.

La dynamique des droits de propriété se trouve également au cœur de la gestion des relations entre les acteurs des réseaux de l'intégration système. L'observation de programmes de codéveloppement technologique procure dans le même temps des indications sur la gestion des contributions logicielles dans les réseaux d'intégration de systèmes et sur la gouvernance des décisions qui les accompagnent. Deux exemples ${ }^{7}$ peuvent être mentionnés ici. Le premier concerne Boeing, qui a utilisé des solutions "libres" pour pointer du doigt des faiblesses [" holes"] dans les services proposés par IBM et Microsoft. L'intégrateur de systèmes conserve ainsi son leadership sur le réseau d'intégration, et en particulier introduit par ce biais des mécanismes d'incitations supplémentaires pour maintenir sa position envers des acteurs qui la contesteraient en cherchant à s'échapper du réseau, ou à ne pas y assumer leur rôle. S'agissant avec les développeurs de logiciels libres de communautés d'acteurs particuliers et de petites entreprises fondées sur des modèles d'affaires réactifs, l'issue de cette démarche stratégique n'est défavorable ni pour les nouveaux entrants (à nouveau par le biais de l'effet de signal), ni pour le réseau d'intégration (qui tire bénéfice de cette nouvelle compétence), ni pour les acteurs industriels "rappelés à l'ordre " (qui peuvent y lire des signaux que le marché ne leur a pas apportés). Cette démarche s'applique à de nombreux cas dans le secteur aéronautique, spatial et Défense, car les acteurs du logiciel libre peuvent non seulement procurer mais aussi adapter de solutions qui existent au moment où le besoin est identifié. Le cas de Certipath décrit dans la section précédente l'illustre également. Les éléments distinctifs prônant la mise en place de solutions libres par les réseaux d'intégration sont

7 Certains éléments sont retirés de l'interview d'un architecte de programme en poste chez un industriel intégrateur, réalisé pendant le workshop FLOSS 1.5 organisé par les auteurs à Salon de Provence en avril 2007. Les commentaires repris ici n'engagent que les seuls auteurs.

80 Economie et Institutions $-\mathrm{n}^{\circ} 12 \& 13-2008-2009$ 
directement associés à la facilité de leur mise en place et à la réactivité des communautés ou des entreprises qui les rendent disponibles, ce dernier argument étant un point majeur retiré des interviews réalisés sur le sujet par les auteurs. Les interviews soulignent en particulier la complexité des relations contractuelles dans les programmes d'armement et l'incapacité à régler facilement et rapidement les questions de droits de propriété intellectuelle. Pour peu que les réglementations en matière de programmes d'armement en coopération soient respectées, le recours à des solutions logicielles libres procure alors une option qui permet de réduire les coûts de transaction au sein du réseau d'intégration et les coûts d'opportunité qui sont directement le miroir des contraintes associées à la gestion du temps au niveau des programmes complexes. On touche ici un point important qui explique pourquoi Certipath et TSCP sont développés sur la base de logiciels libres, et dans une stratégie d'ouverture et de transparence pour tous les acteurs qui contribuent ou participent au projet. Dans les deux perspectives évoquées ici, la question du logiciel libre n'est pas introduite dans le réseau d'intégration système par l'Etat (dans les trois fonctions mentionnées supra au titre des fonctions régaliennes de Défense) mais par l'industrie. Le plus souvent, c'est l'intégrateur de systèmes qui suscite cette opportunité pour servir ses propres objectifs au niveau du respect des spécifications fonctionnelles et des échéances associées au programme.

Le raisonnement sur les droits de propriété intellectuelle renvoie donc, une nouvelle fois, à la gouvernance des relations entre l'Etat, l'intégrateur de systèmes, les autres industriels et les acteurs des communautés qui contribuent au développement et à la production des systèmes d'armes. C'est un sujet largement développé dans la littérature autour de deux aspects précis. D'une part la sélection par une organisation participante de ce qui doit être diffusé hors de ses frontières de ce qui ne doit pas l'être (Harhoff et al., 2003; Henkel, 2006). D'autre part, l'identification des règles d'échange permettant de favoriser la fluidité des transferts des biens logiciels. Qui détient la gouvernance ? Où se situent les acteurs clés ? Les développements précédents ont déjà signalé le rôle majeur de l'intégrateur de systèmes, mais son intervention ne peut pas reposer uniquement sur une logique de propositions spontanées émanant des acteurs innovants autour du logiciel libre. S'agissant de programmes à vocation militaire, il semble peu probable que les informations puissent émaner du réseau en temps et en heure pour susciter "spontanément " de telles offres. Il importe donc de repérer les mécanismes par lesquels des acteurs particuliers vont jouer le rôle d'intermédiaires et développer des capacités permettant d'identifier les sources d'innovation utiles à la concrétisation du projet technologique. Les travaux réalisés par ailleurs sur les

81 Economie et Institutions - n $12 \& 13-2008-2009$ 
mécanismes de transfert de connaissances au sein des réseaux d'intégration suggèrent que cette démarche reposent de façon privilégiée sur les compétences détenues par les intégrateurs. Bien plus que la dynamique de gestion des droits de propriété intellectuelle, c'est alors la problématique associée à l'analyse des capacités d'absorption par chacun des acteurs qui prime (Brown et Duguid, 2001 ; Amin et Cohendet, 2004 ; Mérindol, 2009a).

\section{Hybridité des modèles et gouvernance}

Les acteurs décrits dans les interactions liées à la $R \& D$ et à la production des programmes de Défense dessinent donc un modèle hybride, qui rend compte de l'hétérogénéité de leurs contributions, de leurs motivations et de leurs rôles au sein des réseaux. Toute la difficulté à faire se rejoindre les littératures sur les trois domaines que représentent l'intégration des systèmes, l'innovation ouverte et les logiciels libres provient de ce que les connaissances et les outputs $\mathrm{du}$ processus d'innovation se positionnent différemment au regard du statut privé ou public de la connaissance et des biens issus du processus de l'innovation. Le caractère "hybride " des modèles proposés repose sur la confrontation entre des intérêts privés et publics, marchands et non marchands, ouverts et fermés. Il est non seulement possible de dépasser certaines des antinomies proposées en inscrivant l'ensemble des acteurs de chaque cas d' "hybridité " dans un développement technologique de type collaboratif, mais également d'associer chaque modèle associer à certains niveaux d'efficacité comme cela a été démontré dans le cadre des logiciels libres (Muffato, 2004). Concilier activités non-marchandes et marchandes devient le pré-requis indispensable pour que chaque acteur puisse alors trouver une motivation à sa contribution aux réseaux et aux communautés.

Dans le cadre des dynamiques de l'innovation ouverte et des logiciels open source, les actifs de connaissance revêtent un statut public propice à générer des externalités positives dans d'autres domaines. Dans le cadre des communautés de logiciels open source, les acteurs choisissent souvent de s'engager parce qu'ils adhèrent à un modèle d'organisation où la connaissance et les biens issus des communautés se caractérisent par un statut collectif et public. Allant parfois jusqu'à des motivations socio-idéologiques (Stallman, 2002 ; Weber, 2004), ces communautés voient souvent dans la diffusion de l'innovation un véritable enjeu justifiant les engagements individuels. La mobilisation de communautés du logiciel libre dans le cadre de programmes d'armement conduit alors à prendre en compte certaines incohérences et à souligner des conflits d'intérêt potentiels (Le Texier et Versailles, 2009). Dans le contexte de l'innovation ouverte, le même phénomène se traduit par une volonté de diffusion 
des actifs de connaissance garantis par des droits de propriété intellectuelle, même si ces derniers demeurent privés et soumis à une logique marchande. La différence majeure entre les modèles d'innovation ouverte et fermée porte sur la volonté de diffuser (sous des formes marchandes) les connaissances privées, alors que la stratégie ordinaire des firmes schumpetériennes consiste à se protéger. Les actifs de connaissance, en particulier présentés sous la forme codifiée et échangeable du brevet, y étaient conservés jalousement au sein de la firme de sorte à protéger sa compétitivité dans la dynamique de la concurrence. Avec l'innovation ouverte, la codification sert la volonté de diffusion et le brevet devient même une ressource marchande. En tout état de cause, la position compétitive de la firme n'est plus associée à une position de repli sur soi pour se protéger de la concurrence, mais à une démarche proactive dans les réseaux de connaissance où sa compétitivité se trouve justement associée à l'effet d'entraînement qu'elle suscite dans les réseaux où se créent la connaissance et la valeur.

Reste à développer une investigation du régime de fonctionnement des programmes relatifs aux missions régaliennes de Défense et de Sécurité, où les relations entre le maître d'ouvrage, l'intégrateur de systèmes, les autres acteurs industriels et les communautés associées aux projets sont ancrées dans un mode de travail hiérarchique régulant aussi la répartition des informations. Paradoxalement, les modalités de coordination et d'incitation y sont identiques à ce qui existe dans les communautés du logiciel libre. Lorenzoni et Lipparini (1999) ont montré comment le développement mutuel de compétences relationnelles permet d'y réconcilier des intérêts divergents tout en privilégiant des effets de signalement au sein des réseaux. La divergence profonde entre les deux démarches surgit avec la relation à la notion de bien public. A leur niveau, les biens logiciels qui sont gérés dans une dynamique "open source " revêtent des propriétés similaires aux bien public (Lerner et Tiriole, 2002) parce qu'ils respectent les axiomes de non-rivalité (Baldwin et Clark, 2006) et de non-exclusion (Osterloh et Rota, 2007). C'est la nature publique du bien logiciel libre qui se trouve tout à la fois l'origine des espérances et des craintes des acteurs contributeurs à ce type de communautés. Espérances, car la division du travail et des connaissances sur laquelle s'appuie l'activité du "libre " est propice à l'accroissement de la production. Craintes, car celle-ci implique en même temps un accroissement des coûts de coordination qui peut nuire à la réalisation du projet (Kogut et Zander, 1996) ou parce qu'elle suppose de se coordonner via un acteur particulier qui peut devenir l'acteur structurant (hiérarchique) au sein d'un réseau. Dans les communautés du "libre ", c'est la connaissance qui représente un bien public et un bien collectif à l'échelle de la communauté. 
Dans le cadre des programmes de Défense et de Sécurité, c'est en revanche la mission à laquelle contribue le processus d'innovation qui représente le bien public (pur) (Sandler et Hartley, 1995: 19-20, 43). La dynamique de l'innovation s'y accommode volontiers d'une logique marchande, guidée par les intérêts privés au service d'une mission de service public. Aux Etats-Unis, les relations étroites entre l'état fédéral et les industriels de la Défense ont toujours été développées sur ces bases. Dans les pays européens, les évolutions récentes associées aux restructurations de Défense ont d'ailleurs accentué cette situation en sortant progressivement de la logique d'arsenal. Dans ce cas, la connaissance est privée, cantonnée au sein du réseau d'intégration car sa diffusion large (y compris dans une politique publique valorisant la dualité) conduit à remettre en cause une supériorité tactique ou stratégique qui doit être préservée. Ces considérations sont cohérentes avec les conclusions de West et Gallagher (2003: 102-3), qui les relient plus généralement aux difficultés d'appropriation des solutions technologiques innovantes disponibles au sein des communautés. Ils soulignent en particulier que le caractère marchant ne porte pas sur le logiciel libre en tant que tel, mais sur le service global au service duquel le logiciel libre est mobilisé. Ils mentionnent également que la motivation des acteurs de ces communautés doit susciter des actions spécifiques pour inscrire dans la durée la coopération avec les firmes. De façon plus générale, ils mentionnent aussi que la gouvernance de tels modèles présente une série de difficultés, manifestes lorsqu'une forme de culture organisationnelle doit émerger à partir d'objectifs initiaux distincts. Les arguments présentés ici permettent de caractériser cette culture organisationnelle en matière de contrôle et de diffusion des connaissances.

Ces divers éléments peuvent être présentés sous la forme d'un tableau comparant les quatre modèles de référence utilisés dans cette analyse. 


\begin{tabular}{|c|c|c|c|c|}
\hline & $\begin{array}{c}\text { Modèle } \\
\text { schumpetérien }\end{array}$ & $\begin{array}{c}\text { Réseaux } \\
\text { d'intégration } \\
\text { de systèmes }\end{array}$ & $\begin{array}{c}\text { Innovation } \\
\text { ouverte }\end{array}$ & $\begin{array}{c}\text { Développement } \\
\text { logiciel libre }\end{array}$ \\
\hline $\begin{array}{c}\text { Statut de la } \\
\text { connaissance }\end{array}$ & Privé & $\begin{array}{c}\text { Collectif } \\
\text { (Réseau) sans } \\
\text { diffusion } \\
\text { et Privé } \\
\text { (modules) }\end{array}$ & $\begin{array}{l}\text { Public (diffusion) } \\
\text { Privé (DPI) }\end{array}$ & $\begin{array}{c}\text { Collectif } \\
\text { (communauté) } \\
\text { avec diffusion } \\
\text { et Public }\end{array}$ \\
\hline $\begin{array}{l}\text { Objectif des } \\
\text { brevets }\end{array}$ & Protection & $\begin{array}{l}\text { Protection et } \\
\text { signalement }\end{array}$ & $\begin{array}{l}\text { Créer une } \\
\text { ressource } \\
\text { marchande }\end{array}$ & $\begin{array}{c}\text { Aucun brevet, } \\
\text { mais effet de } \\
\text { signalement }\end{array}$ \\
\hline $\begin{array}{c}\text { Statut des } \\
\text { biens issus } \\
\text { de } \\
\text { l'innovation }\end{array}$ & Privé & $\begin{array}{c}\text { Public pur } \\
\text { pour le } \\
\text { système de } \\
\text { Défense (privé } \\
\text { pour les } \\
\text { composants et } \\
\text { modules) }\end{array}$ & Privé & Public \\
\hline $\begin{array}{l}\text { Motivation et } \\
\text { stratégie des } \\
\text { acteurs }\end{array}$ & $\begin{array}{c}\text { Protéger sa } \\
\text { trajectoire de } \\
\text { développement }\end{array}$ & $\begin{array}{l}\text { L'intégrateur } \\
\text { arbitre } 8 \text { et } \\
\text { répartit les } \\
\text { connaissances } \\
\text { collectives }\end{array}$ & $\begin{array}{c}\text { Créer des actifs } \\
\text { (DPI) de } \\
\text { connaissance } \\
\text { marchandisables }\end{array}$ & Convaincre \\
\hline
\end{tabular}

Tableau 1. Grille comparative des modèles d'analyse

La porosité des frontières des diverses firmes et communautés mobilisées dans cette comparaison dépend de façon directe de deux arguments : la stabilité des mécanismes de transfert de connaissance (et donc la stabilité de son statut public ou privé), et la stabilité des mécanismes d'incitation inhérents aux relations au sein des communautés. Cela concerne à la fois des questions de principes (par exemple : diffuser ou non) et de modalités (à quelle échelle ?). Aucun des modèles n'est totalement étanche aux autres, sauf à rencontrer des acteurs qui oublient les aspects pragmatiques de la vie quotidienne des communautés servant la dynamique de l'innovation ou des programmes d'armement pour s'arcbouter sur des questions de principes. En réalité, la nature-même du logiciel se mettant au service d'un projet plus large (plus complexe et plus intégré) conduit à considérer un modèle de co-développement technologique où les compétences et les actifs de connaissance se mettent au service les uns des autres dans l'élaboration du projet. Dans ce cadre, aucune accumulation désorganisée des compétences techniques n'est envisageable. La grille organisationnelle qui se met en place oriente les contributeurs à partir de leurs compétences

8 Versailles et Mérindol (2009) expliquent que la stratégie de l'intégrateur repose sur l'organisation des connaissances collectives au sein du réseau d'intégration, à partir d'une décision initiale de conserver certaines connaissances en interne.

85 Economie et Institutions $-\mathrm{n}^{\circ} 12 \& 13-2008-2009$ 
relationnelles et techniques. Si elle s'installe dans le temps long, la grille organisationnelle découle, d'une part, de logiques de complémentarités matérialisées dans les architectures industrielles (Jacobides et al., 2006) et, d'autre part, de la mobilité des actifs entre acteurs du réseau constitué des firmes et des communautés. S'agissant de l'interaction qui se développe avec les firmes du réseau d'intégration, les diverses communautés s'inscrivent elles-aussi dans les fondamentaux qui permettent de raisonner sur les avantages compétitifs du produit proposé au client final (ici la Défense dans sa fonction militaire). Toute l'ambition des mécanismes relatifs à l'innovation ouverte ou aux logiciels libres réside donc dans la possibilité de trouver le moyen de concilier les contraintes de chaque acteur avec les frontières du réseau d'intégration, et avec la stratégie des firmes qui le structurent. Pour peu que tous les phénomènes associés à la dynamique de l'innovation demeurent à l'intérieur du réseau d'intégration, peu importe en revanche que la $R \& D$ des firmes soit intégrée ou externalisée puisque les communautés viendront les suppléer dans cette démarche.

La gouvernance des politiques publiques associées à chacun des régimes présentés dans le tableau supra devient alors plus simple à présenter aux termes de cette contribution, car elle dépendra directement des deux variables présentées au paragraphe précédent. Pour les politiques publiques, au niveau national comme européen, l'enjeu principal découle de la conciliation de la variété des attentes des acteurs. Ici se trouvent les défauts de coordination potentiels. Les conflits d'intérêts irréconciliables doivent être d'autant plus évités qu'ils risquent d'introduire des ruptures de capacité et de remettre en cause la continuité du service de Défense et de Sécurité. Il semble peu probable que les programmes de Défense et de Sécurité puissent se dérouler sans la présence d'un acteur de référence, qui a émergé après les restructurations engagées au niveau international sous la forme des firmes intégrateurs de systèmes. Ceux-ci assument des missions qui avaient parfois été dévolues aux Etats. Le rôle de ces firmes ne pouvant être remise en cause pour la gestion des programmes d'armement, il convient alors de considérer comment les politiques publiques peuvent favoriser la coexistence avec les communautés issues de l'open innovation et du logiciel "libre ". Les tensions entre les modèles présentés dans cette contribution se retrouvent, à nouveau, sous la forme d'un choix à réaliser entre les politiques publiques en faveur des transferts de technologie (politique de diffusion) et celles qui privilégient la supériorité technologique (politique de mission). Cette seconde option, retenue sans ambiguïté par les Etats-Unis, conduit à établir des frontières étanches autour du réseau d'intégration puisque la supériorité opérationnelle sur le champ de bataille se trouve alors le prolongement naturel de la supériorité technologique acquise dans le réseau d'intégration. La 
première option traduit le plus souvent les choix réalisés au niveau des structures de la Commission européenne, ce qui se traduit concrètement par les dispositions en faveur de la diffusion des technologies entre les sphères militaires et civiles (politiques de dualité). La liaison entre supériorité opérationnelle et supériorité technologique s'y trouve diluée dans des variables et des relations bien plus nombreuses que dans le modèle retenu aux Etats-Unis et, donc, plus difficile à piloter.

\section{Conclusion}

Cette contribution visait à analyser les formes d'hybridité qui ont émergé entre les acteurs publics et privés, marchands et nonmarchands (y compris communautaires) dans le secteur de l'aéronautique, du spatial et de la Défense depuis une dizaine d'années. Il en ressort pour l'essentiel que la logique actuelle de gouvernance des programmes de Défense et de Sécurité repose aujourd'hui sur des mécanismes qui articulent efficacement les statuts privés et publics de la connaissance au sein des réseaux d'innovation. En résultent des situations où la connaissance est gérée dans des dynamiques collectives, avec des nuances toutefois pour chacune des trois situations considérées. La diffusion des connaissances reste limitée au sein des frontières du réseau d'intégration, dans un cadre où l'intégrateur de systèmes organise la répartition des actifs de connaissance parmi les acteurs. Dans les modèles liés à l'innovation ouverte et aux communautés des logiciels libres, la logique de communautés conduit à ouvrir largement la diffusion des actifs de connaissance, même si les deux situations considèrent de façons très différentes les droits de propriété intellectuelle. Face à ces statuts différents de la connaissance, face aux situations spécifiques que rencontrent les droits de propriété intellectuelle, et compte tenu des statuts des biens issus de l'innovation ou des stratégies propres à chaque famille d'acteurs, des politiques publiques spécifiques peuvent être mises en place pour favoriser la poursuite de la dynamique de l'innovation. Celles-ci doivent s'adapter à chaque cas de figure, ce qui représente en soi une recherche spécifique ouverte à d'autres problèmes. Ceux-ci couvrent en particulier l'articulation entre les politiques publiques de science et de technologie, mal ou peu articulées en Europe avec les politiques d'innovation pour ce qui concerne les questions de Défense et de Sécurité. Comme Mérindol (2009b) le développe pour le rôle de l'Etat au regard des mécanismes d'open science dans la Défense, il convient alors de s'interroger sur la convergence entre, d'une part, les mécanismes d'innovation ouverte ou les dynamiques de communautés, les logiques d'intégration de systèmes, et, d'autre part, les politiques de science et de technologie.

87 Economie et Institutions - n²12\&13-2008-2009 


\section{References}

Amin, A., et Cohendet, P., 2000, Organizational learning and governance through embedded practices, Journal of Management and Governance, 4(2), 93-116.

Amin, A., et Cohendet, P., 2004, Architectures of knowledge: firms, capabilities and communities, New York: Oxford university press.

Azoulay, P., 2004. Capturing knowledge within and across firm boundaries: evidence from clinical development. American Economic Review 94(5), 1591-1612.

Baldwin, C., Clark, K., 2006. The architecture of participation: does code architecture mitigate free riding in the open source development model. Management Science 52(7), 1116-1127.

Brown, J.S., Duguid, P., 1991. Organizational learning and communities of practice: toward a unified view of working, learning and innovation. Organization Science 2(1), 90-111.

Brusoni, S., Prencipe A., 2006, Making Design Rules: A multidomain Perspective, Organization science, 17, pp 179.189.

Brusoni, S., Prencipe A. and Pavitt K., 2001, Knowledge Specialization, Organizational Coupling, and the Boundaries of the Firm: Why Do Firms Know More Than They Make?, Administrative Science Quarterly, 46(4):597-621.

Callon, M., 1999, Le réseau comme forme émergente et comme modalité de coordination: le cas des interactions stratégiques entre firmes industrielles et laboratoires académiques, in Callon et al., 1999, 13-62.

Callon, M., Cohendet, P., Curien, N., Dalle, J.-M., EymardDuvernay, F., Foray, D., et Schenk, E., 1999, Réseau et coordination, Paris: Economica.

Casadesus-Masanell, R., Ghemawat, P., 2006. Dynamic mixed monopoly: a model motivated by Linux vs. Windows. Management Science 52(7), 1072-1084.

Chesbrough, H., Vanhaverbeke W. et West J. (eds), 2008, Open innovation: researching a new paradigm, Oxford: Oxford university press.

Chesbrough, H.W., 2003. Open Innovation: The New Imperative for Creating and Profiting from Technology. Harvard Business School Press, Cambridge, MA.

Chesbrough, H.W., Rosenbloom, R.S., 2002. The role of the model business in capturing value from innovation: evidence from Xerox corporation's technology spin-off companies. Industrial and Corporate Change 11(3), 529-555.

Cohendet, P., Creplet, F., Dupouët, O., 2001. Organisational innovation, communities of practice and epistemic communities: the case of Linux. In: Kirman, A., Zimmermann, J.B. (Eds.), Economics 
with Heterogeneous Interacting Agents. Springer, Berlin, Germany, pp. 303-326.

Dahlander, L., 2007. Penguin in a new suit: a tale of how de novo entrants emerged to harness free and open source software communities. Industrial and Corporate Change 16(5), 913-943.

Dahlander, L., Magnusson, M., 2008. How do Firms Make Use of Open Source Communities? Long Range Planning 41(6), 629649.

David, P. A., 2004. Understanding the energence of 'open science' institutions: functionalist economics in historical context. Industrial and Corporate Change 13(4), 571-589.

Dombrowski, P., Gholz, E., \& Ross, A. L. (2002). Military transformation and the Defense industry after next, The Defense industrial implications of Network Centric Warfare. Newport papers \# 18, New Port (RI): Naval war college.

Dosi, G., Llérena, P., et Sylos Labini, M., 2006, The relationships between science, technologies and their industrial exploitation: An illustration through the myths and realities of the "European Paradoxon", Research Policy, 35, 1450-1464.

Dussauge, P., et Cornu C., 1998, L'industrie française de l'armement, Paris : Economica.

Economides, N., Katsamakas, E., 2006. Two-sided competition of proprietary vs. open source technology platforms and the implications for the software industry. Management Science 52(7), 1057-1071.

Flowers, S., 2008. Harnessing the hackers: the emergence and exploitation of outlaw innovation. Research Policy 37(2), 177193.

Foray, D., 2004. The Economics of Knowledge. The MIT Press, Cambridge, MA.

Gholz, E. (2003). Systems integration in the US Defence industry: who does it and why is it important?. In Prencipe A., A. Davies \& M. Hobday (eds) (pp. 279-306).

Graham, S.J.H., Mowery, D.C., 2008. The Use of Intellectual Property in Software: Implications for Open Innovation. In: Chesbrough, H.., Vanhaverbeke, W., West, J. (Eds.), Open Innovation: Researching a New Paradigm. Oxford University Press, New York, NY, pp. 184-203.

Guesnerie, R., Tirole, J., 1985. L'économie de la recherchedéveloppement : introduction à certains travaux théoriques. Revue Economique 36(5), 843-871.

Hardin, G., 1968. The tragedy of the commons. Science 162(3859), 1243-1248.

Harhoff, D., Henkel, J., von Hippel, E., 2003. Profiting from voluntary information spillovers: how users benfit by freely revealing their innovations. Research Policy 32(10), 1753-1769. 
Henderson, R.M., Clark, K.B., 1990. Architectural innovation: the reconfiguration of existing product technologies and the failure of established firms. Administrative Science Quarterly 35(1), 9-30.

Henkel, J., 2006. Selective revealing in open innovation processes: the case of embedded Linux. Research Policy 35(7), 953969.

Hobday, M., 1998. Product complexity, innovation and industrial organisation. Research Policy 26(6), 689-710.

Hobday, M., Rush, H., Bessant, J., 2004. Approaching the innovation frontier in Korea: the transition phase to leadership. Research Policy 33(10), 1433-1457.

Howells, J., 1996. Tacit knowledge, innovation and technology transfer. Technology Analysis and Strategic Management 8(2), 91-106.

Jacobides, M.G., Knudsen, T., Augier, M., 2006. Benefiting from innovation: value appropriation and the role of industry architectures. Research Policy 35(8), 1200-1221.

Kogut, B., Zander, U., 1996. What Firms Do? Coordination, Identity, and Learning. Organization Science 7(5), 502-518.

Le Texier, Th., \& Versailles, D.W., 2009 (sous presse), Open source software governance serving technological agility The case of open source software within the DoD. International Journal of Open Source Software \& Processes, 1(2), 14-27 (April-June).

Lerner, J., Tirole, J., 2002. Some simple economics of open source. Journal of Industrial Economics 50(2), 197-234.

Lorenzoni, G., Lipparini, A., 1999. The leveraging of interfirms relationships as a distinctive organizational capability: a longitudinal study. Stategic Management Journal 20(4), 317-338.

Mérindol, V. \& Versailles D. W. (2007), International comparisons in the tryptich Government-Industry-Academic research in Defense-related $R \& D$ projects: the crucial role of expertise abilities., chapitre 10 (137-56) in Sherif, M.H., et Khalil, M. (eds), Management of technology: New directions in technology management, Elsevier

Mérindol, V., 2009a, Gestion des compétences et gouvernance de l'innovation: la Défense dans l'économie fondée sur la connaissance. Paris: Economica (collection Recherche en gestion).

Mérindol, V., 2009b, Action publique et $R \& D$ de Défense: une comparaison France - Etats-Unis - Royaume-Uni [titre provisoire] Paris: Dalloz (collection Présage).

Merton, R.K., 1973. The Sociology of Science: Theoretical and Empirical Investigations. University of Chicago Press, Chicago, IL.

Muffatto, M., 2004. Open Source: A multidisciplinary approach. Imperial College Press, London, UK.

O'Sullivan, A., 2006, Why Tense, Unstable and Diverse Relations are Inherent in CO-designing With Suppliers : an 
Aerospace Case Study, Industrial And Corporate Change 15(2): 221250

Osterloh, M., Rota, S., 2007. Open source software development - Just another case of collective invention? Research Policy 36(2), 157-171.

Pavitt, K., 2003, Specialization and systems integration, chapter 5 (78-91) in Prencipe Prencipe Andrea, Davies Andrew, Hobday Michael (eds), The Business of Systems Integration, New York: Oxford University Press.

Prencipe, A., Davies, A. \& Hobday, M., (Eds;). (2003). The business of systems integration. Oxford: Oxford university press.

Richardson, G.B., 1972. The organisation of industry. The Economic Journal 82(32), 883-896.

Sandler, T. et Hartley, K., 1995, The Economics of Defense, Cambridge (UK): Cambridge University Press.

Sapolsky, H.M., 2003, Inventing systems integration in Prencipe Andrea, Davies Andrew, Hobday Michael (eds), The Business of Systems Integration, New York: Oxford University Press.

Simcoe, T.S., 2008. The Use of Intellectual Property in Software: Implications for Open Innovation. In: Chesbrough, H.., Vanhaverbeke, W., West, J. (Eds.), Open Innovation: Researching a New Paradigm. Oxford University Press, New York, NY, pp. 184-203.

Stallman, R.M., 2002. Free Software, Free Society: Selected Essays of Richard M. Stallman. Free Software Foundation, Boston, MA.

Teece, D., 1986. Profiting from technological innovation: implications for integration, collaboration, licensing, and public policy. Research Policy 15(6), 285-305.

Toffler, A., 1980. The Third Wave. William Morrow and Co., New York, NY.

Versailles, D. \& Mérindol, V., 2009, System integration in military aeronautics under the scope of semantic, syntactic and pragmatic knowledge [mimeo, Jan-09],

Versailles, D. W., 2003, Le concept de base industrielle et technologique de Défense: Epoques, approches, acteurs, chapitre 1 in Versailles et al., 2003, pp. 9-36.

Versailles, D. W., Mérindol, V., Cardot, P., 2003, Recherche et technologie, enjeux de puissance, Paris: Economica

Versailles, D.W. (2005). La nouvelle gouvernance des programmes de Défense: les relations entre l'Etat et l'industrie. Revue d'économie indutstrielle, 112(4), 83-105.

von Hippel, 1994, Sticky information and the locus of problem-solving: implications for innovation, Management Science, 40(4), 429-439. 
von Hippel, E., von Krogh, G., 2003. Open source software and the 'private-collective' innovation model: issues for Organization Science. Organization Science 14(2), 208-223.

von Hippel, E., 1986. Lead users: a source of nouvel product concepts. Management Science 32 (7), 791-805.

von Hippel, E., 1988. The Sources of Innovation. Oxford University Press, New York, NY.

von Hippel, E., 2005. Democratizing Innovation. MIT Press, Cambridge, MA.

von Krogh, G., von Hippel., E., 2006. The promise of research on open source software. Management Science 52(7), 975-83.

Weber, S., 2004. The Success of Open Source. Harvard University Press, Cambridge, MA.

Wenger, E.C., 1998. Communities of Practice: Learning, Meaning and Identity. Cambridge University Press, New York, NY.

West, Joel, et Gallagher S., 2008, Patterns of open innovation in open source software, pp. 82-107 in Chesbrough et al., 2008. 